\title{
Isoperimetric Phase Transitions of Two-Dimensional Droplets
}

\author{
François Bavaud \\ Department of Mathematics, Heriot-Watt University, EH14 4AS Edinburgh, Scotland
}

\begin{abstract}
We consider two-dimensional assemblies of particles governed by hamiltonians depending on the area and the perimeter of their convex hull. Provided the hamiltonian is quadratically homogeneous in the coordinates, we find an exact formula for the free energy. Phase transitions resulting from the competition between area and perimeter can easily be produced and explicitly dealt with. We illustrate those features by a simple example undergoing a second-order transition.
\end{abstract}

\section{Introduction}

The competition between the area and the perimeter constitutes, as attested, e.g. by Queen Dido's problem or isoperimetric and Bonnesen inequalities $[1,2]$, one of the central themes in the development of two-dimensional geometry. To be able to exploit this competition in statistical mechanics, we have to find a way to:

i) generate an ensemble of shapes together with an a priori distribution maximizing the entropy

ii) assign an energy to each individual shape.

In two recent publications $[3,4]$, we have started a systematic investigation of the statistical mechanical behaviour of a two-dimensional assembly of particles, whose interaction potential depends on the convex hull spanned by the particles only. One can think of this approach as an attempt to derive from a purely microscopic basis the distribution function of a fluctuating container in a pressure ensemble. Put in another way, such models seek to describe a drop in equilibrium with a mechanical reservoir in terms of its $2 N \approx 10^{23}$ microscopic degrees of freedom. As done in $[3,4]$, we concentrate on interactions of global geometric type only, the two-body interactions between the particles being discarded.

Our approach differs from mainstream statistical mechanical treatment of interfaces (see e.g. [5-9] and references therein) where macroscopic interfaces 
resulting from local interactions between particles are geometrically characterised (e.g. Wulff construction), whereas we describe in this paper sharp boundaries generated by particles submitted to global interactions. Pressure ensembles constitute here the relevant physical context, and the probabilistic set-up is fairly close to situations encountered in Integral Geometry (see e.g. [10-12] and references therein).

To be more specific, we denote by $K\left(\underline{x}_{N}\right) \equiv \operatorname{conv}\left\{x_{1}, \ldots, x_{N}\right\}$ the convex hull spanned by the particles $\left\{x_{1}, \ldots, x_{N}\right\} \equiv \underline{x}_{N} \in R^{2 N}$, and by $H(K)$ the (N-body) hamiltonian. The free energy of the system

$$
g[H]=\lim _{N \rightarrow \infty} g_{N}[H]=\lim _{N \rightarrow \infty} \frac{-k T}{N} \ln \left(Q_{N}[H] / N !\right)
$$

should be interpreted as a Gibbs free energy. The partition function

$$
Q_{N}[H]=\int_{R^{2 N}} d \underline{x}_{N} \delta\left(x_{1}\right) \exp \left(-\beta H\left(K\left(\underline{x}_{N}\right)\right)\right)
$$

is to be evaluated with one particle held fixed, to prevent divergence resulting from the translational invariance. In [4], we considered the model

$$
H_{1}(\lambda, \mu)=\lambda|K|+\mu \frac{|\partial K|^{2}}{4 \pi},
$$

where $|K|$ and $|\partial K|$ are respectively the area and the perimeter of $K\left(\underline{x}_{N}\right)$; we showed the corresponding free energy to satisfy:

$$
g\left[H_{1}(\lambda, \mu)\right]= \begin{cases}-\infty & \text { for } \mu \leqq 0 \text { or/and } \lambda \leqq-\mu \\ k T \ln (\beta \lambda+\beta \mu) & \text { otherwise }\end{cases}
$$

In this paper, we exploit the competition between area $|K|$ and the perimeter $|\partial K|$ of the droplet $K$ by dealing with the whole class of hamiltonians $H(|K|,|\partial K|)$. We impose however the restriction that the average area $|K|$ should behave in the thermodynamic limit as an extensive quantity. Provided the later exists, a sufficient condition for this to be the case is [3] the hamiltonian $H(|K|,|\partial K|)$ to be positive homogeneous of degree 2 in the coordinates $\left\{\underline{x}_{N}\right\}$, or equivalently:

$$
H(|K|,|\partial K|)=h(\eta)|K|, \quad \text { with } \quad \eta:=\frac{|\partial K|^{2}}{4 \pi|K|} .
$$

Clearly, $\eta \geqq 1$, small values of $\eta$ corresponding to approximately circular drops, with $\eta=1$ iff $K$ is a circle. Our result is the following:

Proposition. Let $g[\mathrm{H}]$ be the free energy per particle in the thermodynamic limit corresponding to the hamiltonian (5), where $h(\eta)$ is a continuous function of $\eta$. Define $\gamma:=\min _{\eta \geqq 1} h(\eta)$. Then:

$$
g[H]=\left\{\begin{array}{ll}
-\infty & \text { if } \lim _{\eta \rightarrow \infty} h(\eta)<\infty \text { or } / \text { and } \gamma \leqq 0 \\
k T \ln (\beta \gamma) & \text { otherwise }
\end{array} .\right.
$$




\section{The Proof}

\subsection{Lower Bound for $g[H]$}

Suppose first $\lim _{\eta \rightarrow \infty} h(\eta)<\infty . h(\eta)$ is then bounded above and $H(K) \leqq c|K|$. But the area term alone is not sufficient to prevent the evaporation of the drop and $g[H]=-\infty$, as given by (4). Suppose now $\lim _{\eta \rightarrow \infty} h(\eta)=\infty$, and define $\bar{h}(\eta)$ as the convex envelope (or closure) of $h(\eta)$ for $\eta \in[1, \infty)$. Let the minimum of $\bar{h}(\eta)$ be attained at $\eta_{0}$ (were there many minimizers present, $\eta_{0}$ should be chosen as the greatest one). Assume $\bar{h}(\eta)$ is differentiable in a neighborhood of $\eta_{0}$. By convexity:

$$
\bar{h}(\eta) \geqq \bar{h}\left(\eta_{0}+\varepsilon\right)+\bar{h}^{\prime}\left(\eta_{0}+\varepsilon\right)\left(\eta-\eta_{0}-\varepsilon\right),
$$

where $\varepsilon$ is a small positive quantity. Then the hamiltonian (5) satisfies:

$$
H(|K|,|\partial K|) \geqq\left\{\bar{h}\left(\eta_{0}+\varepsilon\right)-\left(\eta_{0}+\varepsilon\right) \bar{h}^{\prime}\left(\eta_{0}+\varepsilon\right)\right\}|K|+\bar{h}^{\prime}\left(\eta_{0}+\varepsilon\right) \frac{|\partial K|^{2}}{4 \pi} .
$$

The quantity $\bar{h}^{\prime}(\eta+\varepsilon)$ plays the role of the $\mu$ in (4) and is by construction strictly positive. As far as $\bar{h}\left(\eta_{0}\right)>0$, one can choose $\varepsilon$ small enough for the corresponding $\lambda$ to satisfy $\lambda>-\mu$. We then get from (4):

$$
g[H] \geqq k T \ln \left(\beta\left\{\bar{h}\left(\eta_{0}+\varepsilon\right)+\left(1-\eta_{0}-\varepsilon\right) \bar{h}^{\prime}(\eta+\varepsilon)\right\}\right) .
$$

Performing now the limit $\varepsilon \rightarrow 0$, and taking into account $\bar{h}^{\prime}\left(\eta_{0}\right)=0$ as well as $\bar{h}\left(\eta_{0}\right)=h\left(\eta_{0}\right)=\gamma$ achieves the proof. Were $\bar{h}(\eta)$ not differentiable around $\eta=\eta_{0}$, it would nevertheless as a convex function possess a left and a right derivative, so that by rounding off the corner the above reasoning remains applicable.

\subsection{Upper Bound for $g[\mathrm{H}]$}

The equilibrium total free energy minimises the free energy functional:

$$
G_{N}=\min _{f} \int d \underline{x}_{N} f\left(\underline{x}_{N}\right)\left\{H(K)+\kappa x_{1}^{2}+k T \ln \left(N ! f\left(\underline{x}_{N}\right)\right)\right\}
$$

submitted to the conditions:

$$
\begin{aligned}
f\left(\underline{x}_{N}\right) & \geqq 0, \\
\int d \underline{x}_{N} f\left(\underline{x}_{N}\right) & =1 .
\end{aligned}
$$

We have replaced here the $\delta$-function in (2) by an external harmonic field of strength $\kappa(N)$, where $\kappa(N) \rightarrow \infty$ in the thermodynamic limit. Consider now $M$, a convex body containing the origin, and $\rho_{N}(x)$, a normalised distribution function centered at the origin. We choose the trial function:

$$
f\left(\underline{x}_{N}\right)=\left\{\begin{array}{ll}
\rho_{N}\left(x_{1}\right)|M|^{-(N-1)} & \text { if } x_{2}, \ldots, x_{N} \in M \\
0 & \text { otherwise }
\end{array} .\right.
$$

Then, provided the second moment of $\rho_{N}(x)$ decreases faster than $\kappa(N)^{-1}$, the free 
energy per particle satisfies:

$$
g_{N}[H] \leqq\langle H(|K|,|\partial K|)\rangle_{M}-k T \ln \left(\frac{|M|}{N}\right)-k T+O\left(N^{-1}\right),
$$

where $\langle\ldots\rangle_{M}$ denotes the average with respect to the uniform distribution of $N$ particles in $M$. We have at this stage to distinguish between the shape and the size of $M$. Introduce for that purpose a body of area unity $M_{0}$ containing the origin such that $M$ is the image of $M_{0}$ by a dilatation of factor $\sqrt{s}$, i.e. $|M|=s\left|M_{0}\right|=s$. Beside the area constraint, we require the perimeter of $M_{0}$ to satisfy $\left|\partial M_{0}\right|^{2}=4 \pi \eta_{0}$, where $\eta_{0}$ is as before the minimizer of $h(\eta)$. Defining $K_{0}\left(x_{N}\right)$ as the body obtained from $K\left(\underline{x}_{N}\right)$ by the inverse dilatation $s^{-1 / 2}$, we get the result that the average area and perimeter of $K_{0}$ tend in the thermodynamic limit to the area and perimeter of $M_{0}$. More precisely, as shown by Rényi and Sulanke [13]:

$$
\begin{gathered}
\left\langle\left|K_{0}\left(\underline{x}_{N}\right)\right|\right\rangle_{M_{0}}=\left|M_{0}\right|-O\left(N^{-2 / 3}\right), \\
\left\langle\left|\partial K_{0}\left(\underline{x}_{N}\right)\right|\right\rangle_{M_{0}}=\left|\partial M_{0}\right|-O\left(N^{-2 / 3}\right) .
\end{gathered}
$$

The observable $\eta$ in (5) is of course dilatation invariant. From the continuity of $h$ in $|K|$ and $|\partial K|$, we finally get:

$$
\lim _{N \rightarrow \infty} \frac{1}{|M|}\langle H(|K|,|\partial K|)\rangle_{M}=h\left(\eta_{0}\right) .
$$

It remains to optimize (14) with respect to $s=|M|$. This leads to $h\left(\eta_{0}\right) s=N k T$, and the proof is achieved.

\section{Comments}

It appears from the above proof that the whole statistical weight becomes concentrated into the configurations $K\left(\underline{x}_{N}\right)$ satisfying $|\partial K|^{2} / 4 \pi|K|=\eta_{0}$. This large deviation property is reminiscent of similar behaviours encountered in mean field theories. However, it has here to be emphasised that Proposition (6) constitutes an exact statement characterizing the full $N$-body problem, and results from the conjunction of two (rather fortunate) circumstances: the first is that while the area and the square of the perimeter play a dissimilar role in (3), (the later term only insuring a thermodynamic behaviour), both terms contribute nevertheless in the same way provided the thermodynamic limit exists. The second reason is the appearance of area contributions in both the energy and entropy part of the free energy functional (14).

The constraint $\eta(K)=\eta_{0}$ is clearly satisfied by a non-countable set of shapes, as far as $\eta_{0}>1$, and it would be desirable to characterise among those shapes which ones are actually realised with a non-zero probability. Unfortunately, answering such a question would necessitate finer techniques than available at the present time.

The homogeneity property of the hamiltonian (5) makes the temperature dependence of the thermodynamic quantities trivial; in particular, the average energy is always $\langle H\rangle=N k T$. The phase transitions announced in the title refer therefore to qualitative changes occurring when some parameters $\mathscr{P}$, possibly 
contained in $h(\eta ; \mathscr{P})$ and acting as external fields, are varied. The very generality of the result (6) makes any specific choice of a function $h(\eta ; \mathscr{P})$ appear somewhat arbitrary; for any type of transition and associated critical exponents can be produced by a suitable choice of $h(\eta ; \mathscr{P})$. We shall nevertheless illustrate below our theory by a simple model undergoing a second-order phase transition.

\section{An Example}

Let us consider the model:

$$
H(\mu, \varepsilon, p)=\mu \frac{|\partial K|^{2}}{4 \pi}\left(1+\varepsilon\left(\frac{4 \pi|K|}{|\partial K|^{2}}\right)^{p}\right), \quad \mu>0 .
$$

This model, whose study has actually been the starting point of the present paper, represents a simple variant of (3), where the perimeter term insuring the thermodynamic behaviour is modulated by a shape factor controlling the tendency for the drop to be circular; one can imagine that, for $\varepsilon$ or/and $p$ large enough, the energy will dominate the entropy and force non-circular configurations to be produced. As we will see in a short while, this is indeed the case. Our model fits into the general class (5), with

$$
h(\eta ; \mu, \varepsilon, p)=\mu\left(\eta+\varepsilon(\eta)^{1-p}\right) .
$$

We get from (6) the following result, whose derivation we leave to the reader as an exercise:

$$
g[H]= \begin{cases}-\infty & \text { for } p \geqq 0, \quad \varepsilon \leqq-1 \quad \text { or } \quad p \leqq 0, \quad \varepsilon \leqq 0 \\ k T \ln (\beta \mu r(\varepsilon, p)) & \text { otherwise }\end{cases}
$$

where

$$
r(\varepsilon, p)=\left\{\begin{array}{lll}
1+\varepsilon & \text { for } \varepsilon(p-1) \leqq 1 & (\text { Phase A) } \\
\varepsilon^{1 / p} p(p-1)^{(1 / p)-1} & \text { otherwise } & \text { (Phase B) }
\end{array}\right.
$$

The corresponding phase diagram is drawn in Fig. 1. The high symmetry phase A consists in circular drops $\left(\eta_{0}=1\right)$ of area $|K|=\frac{N k T}{\mu(1+\varepsilon)}$. (The dashed line corresponds to model (3).) In the low symmetry phase B we find non-circular $\left(\eta_{0}=(\varepsilon(p-1))^{1 / p}\right)$ shapes of perimeter $\frac{|\partial K|^{2}}{4 \pi}=\frac{p-1}{p} \frac{N k T}{\mu}$ and area $|K|=$ $\left(\eta_{0}\right)^{-1} \frac{|\partial K|^{2}}{4 \pi}$

A suitable order parameter is for instance $\delta=1-\eta_{0}^{-1}(\varepsilon, p)$. It satisfies by construction $\delta \equiv 0$ in phase $\mathrm{A}$, whereas in phase $\mathrm{B}$ it behaves near the transition line $\varepsilon(p-1)=1$ as $\delta(p)=\left(p-p_{c}\right) /\left(p_{c}\left(p_{c}-1\right)\right)$ for $\varepsilon$ fixed and as $\delta(\varepsilon)=\left(\varepsilon-\varepsilon_{c}\right) /\left(\varepsilon_{c}+1\right)$ for $p$ fixed.

One easily verifies the partial derivatives $g_{\varepsilon}$ and $g_{p}$ to be both continuous across the transition line, whereas the susceptibilities $g_{\varepsilon \varepsilon}, g_{\varepsilon p}$ and $g_{p p}$ undergo a finite 


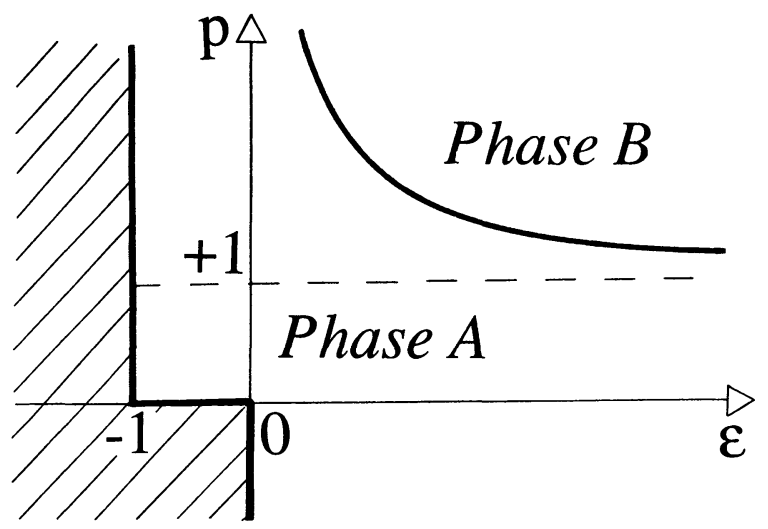

Fig. 1. Phase diagram of (18)

jump, that is to say the system exhibits a second-order phase transition with unity critical exponents.

\section{References}

1. Osserman, R.: Bull. A. M. S. 84, 1182-1238 (1978)

2. Osserman, R.: Am. Math. Monthly 86, 1-29 (1979)

3. Bavaud, F.: J. Stat. Phys. 57, 1059-1068 (1989)

4. Bavaud, F.: Lett. Math. Phys. 20, 75-84 (1990)

5. Zia, R. K. P.: In: Proc. of the XXVIth Scottish Summer School in Physics 1984

6. Rottman, C., Wortis, M.: Phys. Reports 103, 59-79 (1984)

7. Coninck, J. De., Dunlop, F., Rivasseau, V.: Commun. Math. Phys. 121, 401-419 (1989)

8. Pfister, C. E., Penrose, O.: Commun. Math. Phys. 115, 691-699 (1988)

9. Shlosman, S. B.: Commun. Math. Phys. 125, 81-90 (1989)

10. Santaló, L. A.: Integral Geometry and Geometric Probability. Reading, MA: Addison-Wesley 1976

11. Baddeley, A.: Adv. Appl. Prob. 9, 824-860 (1977)

12. Dupain, Y., Kamae, T., Mendès-France, M.: Arch. Rat. Mech. Anal. 94, 155-166 (1986)

13. Rényi, A., Sulanke, R.: Z. Wahrscheinlichkeitstheorie 3, 138-147 (1964)

Communicated by J. Fröhlich

Received December 22, 1989; in revised form March 5, 1990 